\title{
Attenuation of P-Waves by Wave-Induced Fluid Flow
}

S.R. Pride, J.G. Berryman

This article was submitted to Second Biot Conference on Poromechanics, Grenoble, France, August 26-28, 2002

\section{March 29, 2002}

U.S. Department of Energy

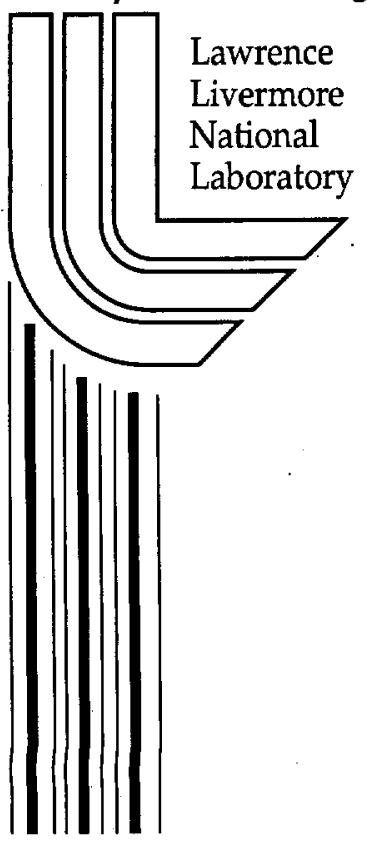




\section{DISCLAIMER}

This document was prepared as an account of work sponsored by an agency of the United States Government. Neither the United States Government nor the University of California nor any of their employees, makes any warranty, express or implied, or assumes any legal liability or responsibility for the accuracy, completeness, or usefulness of any information, apparatus, product, or process disclosed, or represents that its use would not infringe privately owned rights. Reference herein to any specific commercial product, process, or service by trade name, trademark, manufacturer, or otherwise, does not necessarily constitute or imply its endorsement, recommendation, or favoring by the United States Government or the University of California. The views and opinions of authors expressed herein do not necessarily state or reflect those of the United States Government or the University of California, and shall not be used for advertising or product endorsement purposes.

This is a preprint of a paper intended for publication in a journal or proceedings. Since changes may be made before publication, this preprint is made available with the understanding that it will not be cited or reproduced without the permission of the author.

This report has been reproduced directly from the best available copy.

Available electronically at http://www.doe.gov/bridge

Available for a processing fee to U.S. Department of Energy

and its contractors in paper from

U.S. Department of Energy

Office of Scientific and Technical Information

P.O. Box 62

Oak Ridge, TN 37831-0062

Telephone: (865) 576-8401

Facsimile: (865) 576-5728

E-mail: reports@adonis.osti.gov

Available for the sale to the public from

U.S. Department of Commerce

National Technical Information Service

5285 Port Royal Road

Springfield, VA 22161

Telephone: (800) 553-6847

Facsimile: (703) 605-6900

E-mail: orders@ntis.fedworld.gov

Online ordering: http://www.ntis.gov/ordering.htm

OR

Lawrence Livermore National Laboratory

Technical Information Department's Digital Library

http://www.llnl.gov/tid/Library.html 


\title{
Attenuation óf P-waves by wave-induced fluid flow
}

\author{
Steven R. Pride
}

Géosciences Rennes, Université de Rennes 1, Campus Beaulieu, 35042 Rennes Cedex, FRANCE

James G. Berryman

University of California, Lawrence Livermore National Laboratory, P. O. Box 808 L-200, USA

\begin{abstract}
Analytical expressions for three P-wave attenuation mechanisms in rocks are given and numerically compared. The mechanisms are: (1) Biot loss, in which flow occurs at the scale of the wavelength between the peaks and troughs of a $\mathrm{P}$ wave; (2) squirt loss, in which flow occurs at the grain scale between microcracks in the grains and the adjacent pores; and (3) mesoscopic loss, in which flow occurs at intermediate scales between the various lithological bodies that are present in an averaging volume of earth material. Each mechanism is of importance over different frequency bands. Typically, Biot loss is only important at the highest of ultrasonic frequencies (>1 MHz), squirt-loss (when it occurs) is important in the range of $10 \mathrm{kHz}$ to $1 \mathrm{MHz}$, while mesoscale loss dominates at the lower frequencies $(<10 \mathrm{kHz})$ employed in seismology.
\end{abstract}

\section{INTRODUCTION}

In exploration seismology, the material-property resolution required for any forward modeling is dictated by the wavelengths involved and can range anywhere from meters to hundreds of meters depending on the depth of investigation and the seismic source. Averaging elements of earth materials having these dimensions always possess "mesoscopic" heterogeneity in addition to grain-scale heterogeneity. The mesoscopic heterogeneity may be due to variations in lithology (e.g., interbedded sands and shales) or due to macroscopic damage (e.g., highly permeable joints embedded within a much less permeable matrix).

When compressional waves stress such materials, some regions of the material respond with a different fluid pressure than others. The accompanying fluidpressure diffusion attenuates the wave energy. Three types of wave-induced flow may be identified depending on the distance over which the fluid-pressure must equilibrate.

First, there is the wavelength-scale equilibration between the peaks and troughs of the P-wave. This flow is often called "Biot flow" after the seminal work of Biot (1956). The time necessary for the fluidpressure differences between the peaks and troughs to equilibrate goes as $\lambda^{2} / D$ where $\lambda$ is the wavelength and $D$ is pore-pressure diffusivity. For P-waves, the wavelength can be estimated as $\lambda=\sqrt{H / \rho} / f$, where $H$ is the undrained compressional-wave modulus (Biot 1962), $\rho$ is the bulk density of the material, and $f$ is frequency. The diffusivity may be written exactly as $D=M k / \eta$, where $M$ is the fluid storage coefficient defined by Biot (1962), $k$ is the permeability of the material and $\eta$ is the fluid viscosity. The relaxation frequency $f_{c}$, where the Biot-loss per cycle is maximum occurs when the equilibration time equals the wave period

$$
f_{c}=\frac{H}{M} \frac{\eta}{\rho k},
$$

which is also, roughly, the frequency at which viscous-boundary layers develop in the pores of the material [c.f., Eq. (11)]. Thus, at least for Biot loss, the relaxation frequency increases as $\eta / k$.

Second, laboratory samples often have broken contacts between the grains and/or microcracks in the grains themselves. Much of this damage occurs as a rock is brought from depth to the surface. Due to diagenetic processes that heal crack damage, it is uncertain whether in situ rocks in a sedimentary basin ever have significant numbers of broken grain contacts or open microcracks. Nonetheless, if such grainscale damage is present, as it is in lab samples at ambient pressures, the fluid-filled microcracks will respond with greater fluid-pressure deviations than the principle porespace resulting in what Mavko and Nur (1979) have called "squirt flow." Perhaps the most 
complete description of this loss mechanism is that given by Dvorkin et al. (1995). In squirt flow, the time required for a micro-crack of aperature $h$ and length $R$ (roughly a grain size) to equilibrate with an adjacent pore, goes as $R^{2} /\left(h^{2} K_{f} / \eta\right)$ where $K_{f}$ is the fluid's bulk modulus. The frequency at which squirt loss is a maximum is therefore

$$
f_{c}=\left(\frac{h}{R}\right)^{2} \frac{K_{f}}{\eta},
$$

which has just the opposite dependence on $\eta$ as that of the Biot loss, as noted by many authors including Dvorkin et al. (1995). This inverse dependence occurs because the equilibration length $R$ in squirt flow is independent of frequency, while the equilibration length in Biot flow is the wavelength (dependent on frequency).

Finally, there is the "mesoflow" due to the mesoscopic heterogeneity within an averaging volume. More compliant porous materials respond with a greater fluid pressure than do stiffer materials. In the approximation where the mesoscopic heterogeneity can be attributed to just two porous materials, Pride and Berryman (2002) have recently modeled the mesoscopic flow in detail and have obtained general analytical expressions for the frequency dependence of the response. As with squirt flow, the length over which the mesoflow equilibrates is a material property independent of wavelength and so the relaxation frequency is again inversely proportional to the fluid viscosity as in squirt flow.

The goal of the present paper is to give analytical expressions for these three loss processes and to compare them numerically using the unified framework established by Biot.

\section{BIOT DESCRIPTION}

Biot's $(1956 ; 1962)$ theory of the linear acoustics of isotropic porous materials is quite general. It accomodates all three of the above loss mechanisms even though Biot himself did not model the detailed frequency dependence of either the squirt or mesoflow mechanisms; however, he did mention these mechanisms.

With an assumed $e^{-i \omega t}$ time dependence, Biot's poroelastic governing equations can be written

$$
\begin{aligned}
\nabla \cdot \boldsymbol{\tau}= & -\omega^{2}\left(\rho \mathbf{u}+\rho_{f} \mathbf{w}\right) \\
-\nabla p_{f}= & -\omega^{2} \rho_{f} \mathbf{u}-i \omega \frac{\eta}{k(\omega)} \mathbf{w} \\
\boldsymbol{\tau}= & {[(H-4 G / 3) \nabla \cdot \mathbf{u}+C \nabla \cdot \mathbf{w}] \mathbf{I} } \\
& +G\left[\nabla \mathbf{u}+(\nabla \mathbf{u})^{T}-\frac{2}{3} \nabla \cdot \mathbf{u} \mathbf{I}\right] \\
-p_{f}= & C \nabla \cdot \mathbf{u}+M \nabla \cdot \mathbf{w} .
\end{aligned}
$$

The various fields are: $\mathbf{u}$, the average displacement of the solid phase within the averaging volume; $-i \omega \mathbf{w}$, the Darcy filtration velocity; $p_{f}$, the average fluid pressure; and $\tau$, the average stress tensor acting throughout an averaging volume. The Biot elastic moduli $H, C, M$ and $G$ are, in general, complex and frequency dependent even if Biot $(1956,1962)$ did not give specific models for such attenuation and dispersion. These constants are exactly related to the constants having the clearest physical interpretation; namely, the drained bulk modulus $K_{D}$ of the material, the undrained (or "Gassmann") bulk-modulus $K_{U}$, and the Skempton's coefficient $B$ that is defined as the ratio of fluid pressure to confining pressure $p_{c}=-\operatorname{tr}\{\boldsymbol{\tau}\} / 3$ under undrained conditions. These general relations are

$$
\begin{aligned}
H & =K_{U}+4 G / 3 \\
C & =B K_{U} \\
M & =\frac{B^{2}}{1-K_{D} / K_{U}} K_{U} .
\end{aligned}
$$

In the Biot-loss model, $K_{D}, K_{U}$, and $B$ are taken as real constants, while for both the mesoflow and squirt models, the complex frequency dependence of these three constants must be established. In the present paper, the isotropic shear modulus $G$ is taken to be a real constant for all mechanisms since rigorous models of the shear-induced mesoflow and squirt-flow are presently lacking; however, one could use the squirt model of Mavko and Jizba (1991) if an estimate of the frequency dependence of $G$ is desired.

The inertia coefficients are $\rho$, the bulk-material density, and $\rho_{f}$, the fluid density. The coefficient $k(\omega)$ is the complex frequency-dependent dynamic permeability that has been properly modeled by Johnson et al. (1987) as

$$
\frac{k(\omega)}{k_{o}}=\left[\sqrt{1-i \frac{\omega}{\omega_{c}} \frac{4}{n}}-i \frac{\omega}{\omega_{c}}\right]^{-1},
$$

where $k_{o}$ is the d.c. permeability and where the frequency $\omega_{c}$ corresponds to the situation where viscousboundary layers begin to form in the pores. Johnson's model predicts that

$$
\omega_{c}=\frac{1}{F} \frac{\eta}{\rho_{f} k_{o}},
$$

where $F$ is the electrical formation factor that can be estimated (if desired) from Archie's law $1 / F=\phi^{m}$, where $m$ typically lies in the range $[3 / 2,5 / 2]$. Johnson's model further predicts that $n=\Lambda^{2} /\left(k_{o} F\right)$, where $\Lambda$ is a weighted pore-volume to pore-surface ratio; however, as suggested by Johnson et al. (1987), 
we simply take $n=8$ for all materials modeled in this paper.

The P-wave slowness $s$ is determined from the above equations as

$$
s^{2}=b \mp \sqrt{b^{2}-\frac{\rho \tilde{\rho}-\rho_{f}^{2}}{M H-C^{2}}},
$$

where $b=\left(\rho M+\tilde{\rho} H-2 \rho_{f} C\right) /\left[2\left(M H-C^{2}\right)\right]$ is simply an auxilliary parameter and where $\tilde{\rho}=$ $-\eta /[i \omega k(\omega)]$ defines the effective inertia of the fluid in relative motion. Taking the minus sign in Eq. (12) gives an $s$ having an imaginary part much smaller than the real part and that thus corresponds to the normal $\mathrm{P}$-wave. Taking the positive sign gives an $s$ with real and imaginary parts of roughly the same amplitude and that thus corresponds to the slow P-wave (essentially, a pure fluid-pressure diffusion). We are only interested here in the normal P-wave.

The above expressions are used to calculate both the P-wave phase velocity $v_{p}$ and the attenuation measure $Q_{p}^{-1}$ for all three loss mechanisms; namely,

$$
\begin{aligned}
v_{p} & =1 / \operatorname{Re}\{s\} \\
Q_{p}^{-1} & =\operatorname{Im}\{s\} / \operatorname{Re}\{s\} .
\end{aligned}
$$

\section{MESOSCOPIC FLOW}

To estimate the attenuation due to mesoscopic flow, we use the theory of Pride and Berryman (2002) that is rigorously correct whenever the mesoscopic heterogeneity can be attributed to just two porosity types. In such a double-porosity model, the porous material is a composite of two distinct porous continuua. Each porous constituent as well as the overall composite is taken to be isotropic; however, no other geometric or material-property constraints are placed on the mixture. The constituents are labeled with the index $i=$ 1,2 and each has their own suite of Biot coefficients. Specifically, we use $K_{i}$ to denote the drained bulkmodulus of phase $i, B_{i}$ to denote the Skempton's coefficient, and $\alpha_{i}$ to denote the Biot and Willis (1957) parameter that is related to the Gassmann modulus $K_{i}^{u}$ of phase $i$ as $\alpha_{i}=\left(1-K_{i} / K_{i}^{u}\right) / B_{i}$. Each phase has as well its own shear modulus, permeability and formation factor and both phases are saturated by the same fluid. Finally, the volume fraction of each constituent is denoted $v_{i}$ for $i=1,2$, where $v_{1}+v_{2}=1$.

Pride and Berryman (2002) have obtained the effective complex constants $K_{D}, K_{U}$ and $B$ of the com- posite in the form

$$
\begin{aligned}
\frac{1}{K_{D}} & =a_{11}-\frac{a_{13}^{2}}{a_{33}+\gamma / i \omega} \\
\frac{1}{K_{U}} & =a_{11}-a_{22}-\frac{\gamma}{i \omega}-\frac{a_{13}^{2}+\left(a_{23}-\gamma / i \omega\right)^{2}}{a_{33}+\gamma / i \omega} \\
B & =\frac{-a_{12}\left(a_{33}+\gamma / i \omega\right)+a_{13}\left(a_{23}-\gamma / i \omega\right)}{\left(a_{22}+\gamma / i \omega\right)\left(a_{33}+\gamma / i \omega\right)-\left(a_{23}-\gamma / i \omega\right)^{2}}
\end{aligned}
$$

where the geomechanical constants $a_{i j}$ are given by [c.f., Berryman and Pride (2002)]

$$
\begin{aligned}
& a_{11}=\frac{1}{K_{o}} \\
& a_{22}=\frac{v_{1} \alpha_{1}}{K_{1}}\left(\frac{1}{B_{1}}-\frac{\alpha_{1}\left(1-Q_{1}\right)}{1-K_{1} / K_{2}}\right) \\
& a_{33}=\frac{v_{2} \alpha_{2}}{K_{2}}\left(\frac{1}{B_{2}}-\frac{\alpha_{2}\left(1-Q_{2}\right)}{1-K_{2} / K_{1}}\right) \\
& a_{12}=-\frac{v_{1} Q_{1}}{K_{1}} \alpha_{1} \\
& a_{13}=-\frac{v_{2} Q_{2}}{K_{2}} \alpha_{2} \\
& a_{23}=-\frac{\alpha_{1} \alpha_{2} K_{1} / K_{2}}{\left(1-K_{1} / K_{2}\right)^{2}}\left(\frac{1}{K}-\frac{v_{1}}{K_{1}}-\frac{v_{2}}{K_{2}}\right)
\end{aligned}
$$

Here, $K_{o}=\lim _{\omega \rightarrow 0} K_{D}(\omega)$ is the drained bulk modulus of the composite in the low-frequency limit and is the one constant that is not defined in terms of the underlying constituent properties. All dependence of the $a_{i j}$ on the mesoscopic geometry of the constituents and on the underlying shear properties is contained within $K_{o}$. The two constants $Q_{1}$ and $Q_{2}$ are defined

$$
\begin{aligned}
& v_{1} Q_{1}=\frac{1-K_{2} / K_{o}}{1-K_{2} / K_{1}} \\
& v_{2} Q_{2}=\frac{1-K_{1} / K_{o}}{1-K_{1} / K_{2}} .
\end{aligned}
$$

The remaining model parameter is the complex function $\gamma(\omega)$ that has units of inverse viscosity and is given by

$$
\gamma(\omega)=\gamma_{o} \sqrt{1-i \frac{\omega}{\omega_{m}}}
$$

where the mesoscopic flow relaxation frequency $\omega_{m}$ is defined by

$$
\omega_{m}=\frac{\eta B_{1} K_{1}}{k_{1} \alpha_{1}}\left[\left(1+\sqrt{\frac{k_{1} B_{2} K_{2} \alpha_{1}}{k_{2} B_{1} K_{1} \alpha_{2}}}\right) \frac{V}{S} \frac{\gamma_{o}}{\theta}\right]^{2},
$$


and where $\gamma_{o}$ is given by

$$
\begin{aligned}
\gamma_{o}= & \frac{v_{1}}{\eta} \frac{k_{1}}{L_{1}^{2}} \frac{\left(Q_{1}-B_{o} / \beta_{1}-B_{o} / B_{1}\right)}{1-B_{o} / B_{1}} \\
& \times\left[1-\frac{\alpha_{2}}{\alpha_{1}} \frac{1-B_{o} / B_{2}}{1-B_{o} / B_{1}} \frac{K_{1}}{K_{2}} \frac{L_{2}^{2}}{L_{1}^{2}}\right]^{-1} .
\end{aligned}
$$

Here, $B_{o}=\lim _{\omega \rightarrow 0} B(\omega)$ is the low-frequency Skempton's coefficient of the composite given by

$$
B_{o}=-\frac{a_{12}+a_{13}}{a_{22}+2 a_{23}+a_{33}},
$$

while $\beta_{1}$ is defined

$$
\frac{v_{1}}{\beta_{1}}=\frac{v_{1} \alpha_{1}}{K_{1}} \frac{1-Q_{1}}{1 / K_{1}-1 / K_{2}}+\frac{v_{2} \alpha_{2}}{K_{2}} \frac{1-Q_{2}}{1 / K_{2}-1 / K_{1}}
$$

The ratio $V / S$ is the volume of composite divided by the surface area of the interface separating the two constituents within $V$. The dimensionless parameter $\theta$ is given formal definition by Pride and Berryman (2002), but is expected to be close to unity as discussed by the authors. Finally, the lengths $L_{1}$ and $L_{2}$ define the distance over which the fluidpressure gradients are defined in each phase when the fluid-pressure equilibration is almost complete. Pride and Berryman (2002) have rigorously defined these lengths but, due to the complexity of the definitions, they are treated here simply as representing a characteristic linear dimension of each phase.

\section{SQUIRT FLOW}

Here we consider the Dvorkin et al. (1995) squirt-flow model. In this model, the individual grains making up the solid skeleton are taken to be porous. The "grain porosity" is due either to microcracks in the grains themselves or to broken grain contacts that are themselves crack like. Since such crack-like porosity is more compliant than the principle porespace residing between the grains, there develop fluid-pressure differences between the crack porosity of the grains and the principle pores. The fluid-pressure response and subsequent fluid flow inside the porous grains may thus be modeled using Biot theory (poroelasticity).

This general perspective adopted by Dvorkin et al. (1995) is a highly fruitful way of analyzing squirt flow. Two small issues may be raised about the way Dvorkin et al. (1995) develop their specific model: (1) it is unnecessary to assume that the grain space may be divided into effective unit cylinders aligned in the direction of wave propagation and that are immersed in a reservoir of constant fluid pressure; and (2) in taking the limit where the crack porosity is small, these authors assume that the effective drained bulk modulus $K_{D}^{g}$ of the porous grains is independent of the grain porosity $\phi_{g}$ which results in a spurious dependence on the fluid modulus $K_{f}$. Here, for simplicity, we continue to make the assumption of the first issue; however, we attempt to correct the second issue.

The key part of the Dvorkin et al. (1995) model is to obtain an effective complex solid modulus $K_{g}$ of the porous grains that replaces the mineral modulus in the standard Biot theory and to obtain the effective complex drained modulus $K_{D}$. It is assumed that if the cracked grains are somehow healed without changing the topology of the principle porespace, then a socalled "high-pressure" drained modulus $K_{D}^{\mathrm{hp}}$ may be defined with $K_{s}$ now representing the true modulus of the single mineral making up the intact grain space. Dvorkin et al. (1995) correctly state that

$$
\frac{1}{K_{D}}-\frac{1}{K_{g}}=\frac{1}{K_{D}^{\mathrm{hp}}}-\frac{1}{K_{s}}
$$

This equation serves to define $K_{D}$ under the restriction that all grains are made of a single isotropic mineral. Using the cylindrical-grain assumption referred to above, Dvorkin et al. (1995) determine the effective complex mineral modulus of the porous grains to be

$$
K_{g}(\omega)=\frac{K_{D}^{g}+\alpha_{g}^{2} M_{g}[1-f(\omega)]}{1-\alpha_{g} B f(\omega)}
$$

where $K_{D}^{g}$ is the previously mentioned drainedmodulus of a porous grain, $\alpha_{g}$ is the Biot-Willis parameter of a porous grain

$$
\alpha_{g}=1-K_{D}^{g} / K_{s},
$$

and $B$ is the Skempton's coefficient of the singlemineral porous material

$$
B=\frac{1 / K_{D}^{\mathrm{hp}}-1 / K_{s}}{1 / K_{D}^{\mathrm{hp}}-1 / K_{s}+\phi_{p}\left(1 / K_{f}-1 / K_{s}\right)},
$$

with $\phi_{p}$ the porosity of the principle porespace $\left(\phi_{g} \ll\right.$ $\phi_{p}$ ). The Biot storage coefficient $M_{g}$ of the porous grains is defined

$$
M_{g}=\frac{K_{f}}{\phi_{g}} \frac{1}{1+\Delta_{g}}
$$

where $\Delta_{g}$ is the auxiliary term

$$
\Delta_{g}=\frac{1-\phi_{g}}{\phi_{g}} \frac{K_{f}}{K_{s}}\left(1-\frac{K_{D}^{g}}{\left(1-\phi_{g}\right) K_{s}}\right) .
$$

All frequency dependence is confined to the complex function $f(\omega)$ given by

$$
f(\omega)=\frac{2 J_{1}\left(\sqrt{i \omega / \omega_{\mathrm{sq}}}\right)}{\sqrt{i \omega / \omega_{\mathrm{sq}}} J_{0}\left(\sqrt{i \omega / \omega_{\mathrm{sq}}}\right)}
$$




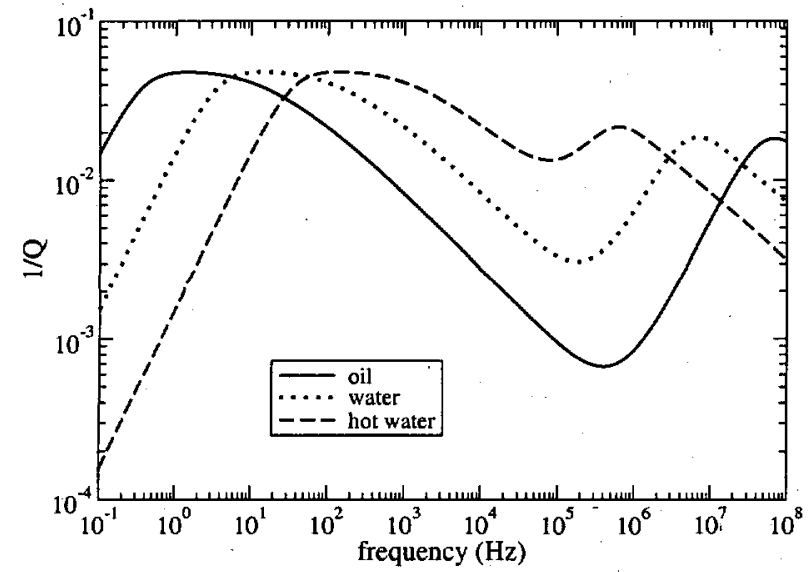

Figure 1: The P-wave attenuation of a double porosity composite having the properties discussed in the text. The three curves correspond to the viscosities of $10^{-2}$ $\mathrm{Pa} \cdot \mathrm{s}$ (oil), $10^{-3} \mathrm{~Pa} \cdot \mathrm{s}$ (ambient water), and $10^{-4} \mathrm{~Pa} \cdot \mathrm{s}$ (hot water).

where $J_{0}$ and $J_{1}$ are Bessel functions and where the relaxation frequency is defined by

$$
\omega_{\mathrm{sq}}=\frac{k_{g}}{R^{2}} \frac{M_{g}}{\eta} .
$$

Here, $k_{g}$ is the permeability of the porous grains and $R$ is a characteristic grain radius.

These choices determine both the effective complex drained modulus $K_{D}(\omega)$ and the real constant $B$. It remains only to state the standard Gassmann (1951) result for the undrained modulus

$$
K_{U}(\omega)=K_{D}(\omega) /[1-\alpha(\omega) B]
$$

where $\alpha(\omega)=1-K_{D}(\omega) / K_{g}(\omega)$. Using these three expressions for $K_{U}, K_{D}$ and $B$ in Eqs. (7)-(9) determines the complex $H, C$ and $M$ of Biot theory, which in turn determines the squirt loss via Eq. (14).

\section{NUMERICAL EXAMPLES}

Some examples of $\mathrm{P}$-wave attenuation are now given. All examples were determined using Eq. (14).and the appropriate $H, C$ and $M$ for the model.

In the mesoscopic flow model, the drained modulus $K_{o}$ of the double-porosity composite is taken to be a harmonic average of the two constituent drained moduli, i.e., $K_{o}=K_{1}^{v_{1}} K_{2}^{v_{2}}$. Phase 2 in the following examples is assumed to be "embedded" within phase 1 so that $L_{1},>L_{2}$, and we assume for all curves that $L_{1}=2 L_{2}$. Because of such embedding, we also make the crude modeling estimates of $V / S=\left(L_{1}+L_{2}\right)^{3} /\left(6 L_{2}^{2}\right), v_{2}=L_{2}^{3} /\left(L_{1}+L_{2}\right)^{3}$, and $v_{1}=1-v_{2}$. These choices formally correspond to phase 2 being little cubes embedded within phase 1 . Although the detailed nature of the function $Q^{-1}(\omega)$ changes depending on how $L_{1} / L_{2}, V / S$, and $v_{2}$ are modeled, our present choice is nevertheless representative of the level of attenuation that is present in

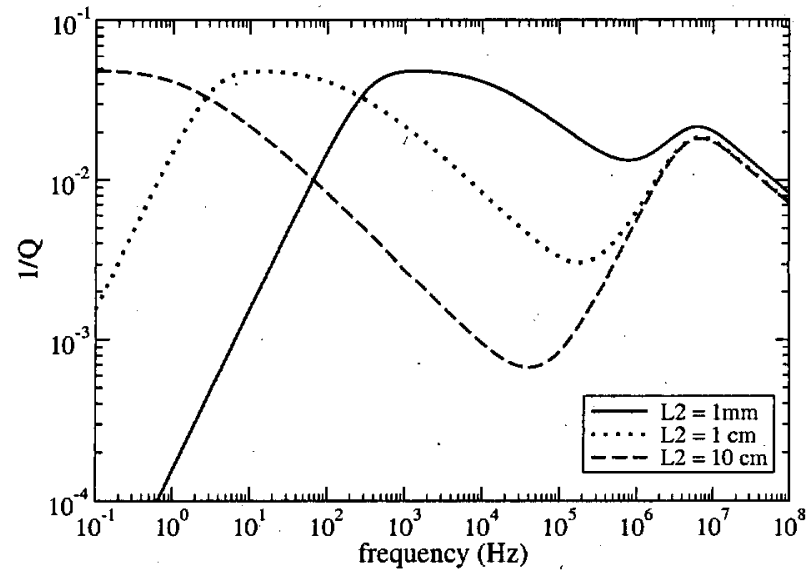

Figure 2: The $\mathrm{P}$-wave attenuation of a double-porosity composite when the size $L_{2}$ of the embedded phase varies at fixed ratio $L_{1} / L_{2}=2$. It is not possible to consider smaller lengths than $1 \mathrm{~mm}$ because the notion of a porous continuum requires at least a few tens of grains to be present in each phase.

double-porosity models. In the limited space here, exhaustive examples are not possible.

We assume that the zero-frequency drained moduli in each phase $K_{i}$ can be determined from the effective-medium formula

$$
K_{i}=\frac{\left(1-\phi_{i}\right) K_{s}}{1+a_{i} \phi_{i}},
$$

where the degree of frame consolidation is controlled by the dimensionless factor $a_{i}$. For all curves, we assume that phase 1 is a well consolidated sandstone and take $\phi_{1}=0.15, a_{1}=10$, and $k_{o 1}=10^{-14} \mathrm{~m}^{2}$ (where $k_{o 1}$ is the d.c. permeability of phase 1). Phase 2 is taken to be a less well-consolidated sand having $\phi_{1}=0.25, a_{2}=200$, and $k_{o 2}=10^{-13} \mathrm{~m}^{2}$. The macroscopic dynamic permeability of the composite is taken to be $k(\omega)=v_{1} k_{1}(\omega)$. Both phases are assumed to be occupied by a single isotropic mineral, so that Skempton's coefficient $B_{i}$ of each phase is determined using Eq. (34) [replace $K_{D}^{\mathrm{hp}}$ and $\phi_{p}$ by $K_{i}$ and $\left.\phi_{i}\right]$, while the Biot-Willis parameter of each phase is determined using $\alpha_{i}=1-K_{i} / K_{s}$.

In the first example given in Fig. 1, we take $L_{2}=1$ $\mathrm{cm}$ and vary the fluid viscosity to show how the two peaks in the attenuation vary. The peak to the left for each curve corresponds to the frequency where the mesoscopic structure just has time to equilibrate in one cycle, while the peak to the right corresponds to the Biot-loss maximum which occurs when the entire wavelength of fluid pressure variation just equilibrates in a cycle. Note that the effect of viscosity is to shift the peaks differently as discussed in the introduction. As expected, increasing fluid viscosity has the effect of shifting the peak in mesoscale loss to lower frequencies, because the fluid-pressure diffusivity is decreasing with viscosity. Because the Biot 
attenuation peak is always visible in these plots as the high-frequency peak, we do not give a separate plot for a simple uniform solid-frame porous material.

In the second example given in Fig. 2, we keep the porous continuum properties the same as in the first example but change the the size $L_{2}$ of the embedded phase while keeping the ratio $L_{1} / L_{2}=2$ constant and using the fixed viscosity of water. Because the amount of material that must equilibrate is independent of frequency, we see that the peak mesoscale loss shifts as the square of the distance as is expected for any diffusion process. Clearly, if several different porosity types were present, and if each porosity type had a different length, one could expect nearly constant $Q$ behavior to emerge over the seismic band of frequencies $\left(f<10^{4} \mathrm{~Hz}\right)$.

To model squirt flow, we need to propose a model for the effective porous grain properties $k_{g}$ (grain permeability) and $K_{D}^{g}$ (drained bulk modulus of a porous grain). Since the porosity within the grains is assumed to be due to microcracks, it is reasonable to take $k_{g}=\phi_{g} h^{2} / 12$ as the permeability model, where $h$ is a representative aperature (or gap) of the microcracks. The grain porosity is necessarily on the order of $\phi_{g}=h / R$, where $R$ is a representative grain radius. Last, the drained modulus of a grain is again taken in the form $K_{D}^{g}=\left(1-\phi_{g}\right) K_{s} /\left(1+a_{g} \phi_{g}\right)$ with $a_{g}=200$ (just like phase 2 of the mesoscopic model), which corresponds to the microcracks being rather compliant. It is important to emphasize that Dvorkin et al. (1995) take both $k_{g}$ and $K_{D}^{g}$ as fitting parameters, independent of the grain porosity, which can lead to rather different numerical results than those given here. We feel that the present modeling is more internally consistent and therefore more physical.

In Fig. 3, we vary the aspect ratio $h / R$ for a "highpressure" frame having a consolidation parameter of $a_{\mathrm{hp}}=10$ and $\phi_{p}=0.15$, just like phase 1 of the mesoscopic model. As required by Eq. (38), the peak squirt loss shifts to lower frequency as the square of the aspect ratio and decreases in amplitude in direct proportion to the aspect ratio. At ultrasonic frequencies, squirt loss may dominate all other mechanisms, especially in lab samples for which many broken grain contacts are expected to be present. However, in the seismic band of frequencies, squirt loss is not expected to be the controlling loss mechanism.

To conclude, we underline that what people typically call "Biot loss" is the pressure equilibration occurring between the peaks and troughs of a compressional wave when the porous material is taken to be uniform over the wavelength. Mesoscopic heterogeneity over the scale of a wavelength can be responsible for considerable low-frequency attenuation. By varying the parameters of the double-porosity model, a wide range of $Q^{-1}(\omega)$ behavior can be obtained. At-

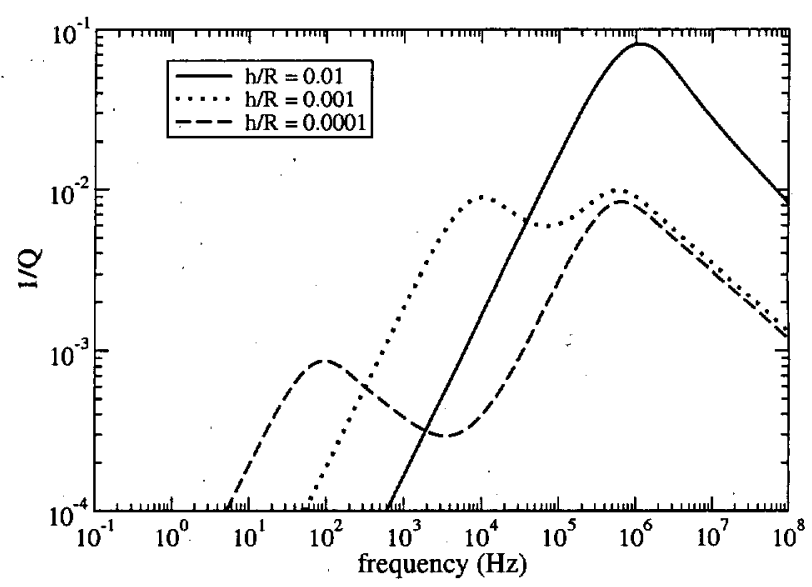

Figure 3: The Dvorkin et al. 1995 squirt model as introduced into Biot theory for three different aspect ratios, where $h$ is an effective micro-crack aperature and $R$ is a grain size.

tenuations can have inverse $Q$ up to $Q^{-1}=1$, if the embedded phase is taken to be extremely compliant.

\section{ACKNOWLEDGMENTS}

Work of JGB was performed under the auspices of the U.S. Department of Energy under contract No. W7405-ENG-48 and supported specifically by the Geosciences Research Program of the DOE Office of Basic Energy Sciences, Division of Chemical Sciences, Geosciences and Biosciences.

\section{REFERENCES}

Berryman, J. G. and S. R. Pride (2002). Models for computing geomechanical constants of doubleporosity materials from the constituents' properties. J. Geophys. Res. 107, 1000-1015.

Biot, M. A. (1956). Theory of propagation of elasti waves in a fluid-saturated porous solid. I. Lowfrequency range. J. Acoust. Soc. Am. 28, 168178.

Biot, M. A. (1962). Mechanics of deformation and acoustic propagation in porous media. J. Appl. Phys. 33, 1482-1498.

Biot, M. A. and D. G. Willis (1957). The elastic coefficients of the theory of consolidation. $J$. Appl. Mech. 24, 594-601.

Dvorkin, J., G. Mavko, and A. Nur (1995). Squirt flow in fully saturated rocks. Geophysics 60, 97-107.

Gassmann, F. (1951). Über die Elastizität poröser Medien. Vierteljahrsschrift der Naturforschenden Gesellschaft in Zürich 96, 1-23.

Johnson, D. L., J. Koplik, and R. Dashen (1987). Theory of dynamic permeability and tortuosity in fluid-saturated porous media. J. Fluid Mech. 176, 379-402. 
Mavko, G. and D. Jizba (1991). Estimating grainscale fluid effects on velocity disperion in rocks. Geophysics 56, 1940-1949.

Mavko, G. and A. Nur (1979). Wave attenuation in partially saturated rocks. Geophysics $44,161-$ 178.

Pride, S. R. and J. G. Berryman (2002). Linear dynamics of double-porosity and dualpermeability materials. submitted.

This work was performed under the auspices of the U.S. Department of Energy by the University of California, Lawrence Livermore National Laboratory under Contract No. W-7405-Eng-48. 